\title{
Práticas de conciliação e satisfação no trabalho: mediação da facilitação do trabalho na família
}

\author{
Work-family facilitation: mediating the relationship between \\ practices of conciliation and job satisfaction
}

\author{
Maria José CHAMBEL' $^{1}$ \\ Marina Veríssimo dos SANTOS
}

\begin{abstract}
Resumo
Este estudo investigou o efeito que a disponibilização de práticas conciliadoras do trabalho e da família por parte das organizações tem na satisfação que os trabalhadores experienciam com o seu trabalho; também analisou o papel que a facilitação do trabalho na família desempenha enquanto mediadora da relação entre estas variáveis. Os dados foram obtidos mediante a participação de 342 sujeitos, 171 casais, casados ou vivendo maritalmente, os quais responderam a um questionário. Os participantes estavam empregados em uma grande variedade de profissões e organizações. Os resultados suportam a hipótese de que a facilitação do trabalho na família medeia a relação entre as práticas de conciliação e a satisfação com o trabalho. Deste modo, os resultados salientam o papel importante que esta facilitação desempenha nas atitudes dos empregados perante as práticas organizacionais conciliadoras do trabalho e da família, apresentando, assim, uma explicação sobre o mecanismo por meio do qual as práticas de conciliação têm impacto positivo na satisfação dos empregados.
\end{abstract}

Unitermos: Facilitação social. Família. Satisfação no emprego.

\begin{abstract}
The present study explored the effect that the availability of conciliatory work and family practices on the part of organizations, has on the job satisfaction experienced by the workers. Moreover, this study examined the role that work-family facilitation plays as a mediator in the relationship between these variables. The data was gathered from 342 subjects, 171 couples, married or cohabiting, who responded to a survey. The respondents were employed in a wide variety of occupations and organizations. The results demonstrated that work-to-family facilitation explains a significant amount of unique variance associated with job satisfaction, over and above the variance explained by the practice of conciliation. The results support the hypothesis that work-to-family facilitation mediates the relationship between the practices of conciliation and job satisfaction. In this way, the results underscore the important role that work-to-family facilitation plays in determining employees' reactions, in terms of job satisfaction, to the practice offered by organizations of reconciling the work and family domains, thereby offering an explanation of the mechanism through which these practices have a positive impact on employee satisfaction.
\end{abstract}

Uniterms: Social facilitation. Family. Job satisfaction.

$\cot$

1 Universidade de Lisboa, Faculdade de Psicologia e de Ciências da Educação. Alameda da Universidade, 1649-013, Lisboa, Portugal. Correspondência para/Correspondence to: M.J. CHAMBEL. E-mail: <mjchambel@fpce.ul.pt>. 
Nas últimas décadas tem-se assistido a grandes mudanças na estrutura das famílias e na composição demográfica da força de trabalho. Alguns exemplos destas mudanças são: o aumento da entrada de mulheres na força de trabalho, sendo que algumas têm filhos pequenos ou outros dependentes; o maior número de pais solteiros que trabalham; o aumento do número de casais em que ambos os membros trabalham (Allen, 2001; Boyar \& Mosley, 2007; Ford, Heinen \& Langkamer, 2007; Grzywacz \& Marks, 2000; Parasuraman \& Greenhaus, 2002). Todas estas alterações têm aumentado a probabilidade de que os empregados de ambos os sexos tenham simultaneamente responsabilidades domésticas e de trabalho (Allen, 2001; Boyar \&Mosley, 2007; Clark, 2001). Assim, estes trabalhadores, ao possuírem responsabilidades nestes dois domínios da vida, deparam-se com um grande desafio: conjugar o seu envolvimento no trabalho e na família (Grzywacz \& Marks, 2000). Não é por isso surpreendente que a atenção dada às questões relacionadas com o trabalho e com a família tenha aumentado ao longo destes anos e continue a aumentar (Ford et al., 2007).

Apesar de a investigação sobre a dinâmica entre o trabalho e a família ter sido dominada pela perspectiva do conflito (Greenhaus, Allen \& Spector, 2006; Perrewe \& Hochwarter, 2001), definido como"uma forma de conflito inter-papel no qual as pressões de papel dos domínios do trabalho e da família são mutuamente incompatíveis em algum aspecto" (Greenhaus \& Beutell, 1985, p.77). Frone (2003) sugeriu que uma boa compreensão da interface trabalho-família deve incluir ambos os componentes: o de conflito (interface negativa) e o de facilitação (interface positiva). Deste modo, o autor articulou um modelo quádruplo de equilíbrio trabalho-família incorporando conflito e facilitação do trabalho na família e da família no trabalho. A facilitação trabalho-família (FTF), de acordo com o autor, é a "extensão para a qual a participação na família (trabalho) torna-se mais fácil em virtude das experiências, aptidões e oportunidades ganhas ou desenvolvidas no trabalho (família)" (p.145), ocorrendo bidirecionalmente: facilitação do trabalho na família (F T $\rightarrow F)$ e facilitação da família no trabalho (FF $\rightarrow$ T) (Wayne, Musisca \& Fleeson, 2004). É de salientar que a $\mathrm{FT} \rightarrow \mathrm{F}$ tem igualmente sido referida como enriquecimento do trabalho na família. De fato, em oposição à perspectiva do conflito, a teoria do enri- quecimento de papel propõe que a participação em múltiplos papéis oferece aos sujeitos um grande número de oportunidades e recursos, os quais podem ser utilizados para promover o crescimento e um melhor funcionamento em outros domínios da vida (Grzywacz $\&$ Marks, 2000). No caso específico F $\rightarrow \rightarrow F$ pressupõe-se que as atitudes, sentimentos, emoções, competências e comportamentos aprendidos e utilizados no trabalho são úteis e enriquecem a vida familiar.

Atualmente, um grande número de organizações demonstra preocupação com aquilo que se passa na vida familiar dos seus empregados, implementando um conjunto de práticas para dar suporte aos trabaIhadores na gestão das suas responsabilidades familiares: as chamadas práticas de conciliação entre o trabalho e a família (Allen, 2001; Shockley \& Allen, 2007; Thomas \& Ganster, 1995; Thompson, Beauvais \& Lyness, 1999). Para as organizações, estas práticas são uma forma de manter vantagem competitiva, aumentando a moral e atraindo e retendo uma força de trabalho dedicada. Para os empregados, estas práticas são uma forma de aliviar as dificuldades inerentes à necessidade de conciliar o seu papel profissional e familiar (Allen, 2001).

Alguns estudos têm verificado que a existência destas práticas de conciliação do trabalho e da família relaciona-se positivamente com a satisfação com o trabalho (Baltes, Briggs, Huff, Wright \& Neuman, 1999; Thomas \& Ganster, 1995). Por outro lado, outros estudos têm mostrado que as práticas de conciliação disponíveis são importantes para os trabalhadores equilibrarem os domínios do trabalho e da família, relacionando-se com a facilitação do trabalho na família (Carlson, Kacmar, Wayne \& Grzywacz, 2006; Grzywacz \& Butler, 2005). Além disto, existe um considerável número de estudos que demonstra a existência de uma relação entre FT $\rightarrow$ F e satisfação com o trabalho (Aryee, Srinivas \&Tan, 2005; Boyar \& Mosley, 2007; Carlson et al., 2006).

Tendo em conta todos os aspectos referidos anteriormente, neste estudo considerou-se que a FT $\rightarrow F$ tinha um efeito mediador na relação entre as práticas de conciliação e a satisfação com o trabalho. Deste modo, teve como objetivos, por um lado, analisar o efeito que as práticas organizacionais conciliadoras dos domínios do trabalho e da família têm na satisfação com o trabalho e, por outro, analisar se a relação entre estas duas variáveis é mediada pela $\mathrm{FT} \rightarrow \mathrm{F}$. 
Do ponto de vista teórico, este estudo permite aumentar os conhecimentos sobre o lado positivo da interface trabalho-família, que se integra no novo paradigma da psicologia positiva, focalizada nos pontos fortes dos indivíduos e dos sistemas sociais (Seligman, 2002). A investigação do conflito entre o trabalho e a família tem predominado, bem como as suas repercussões negativas para o bem-estar dos empregados. No entanto, tem sido salientada a necessidade de se desenvolverem estudos centrados nas vantagens de se acumular estes dois papéis, bem como nas características organizacionais que promovem o desempenho familiar através do desempenho do papel profissional (Greenhaus \& Powell, 2006). Do ponto de vista prático, este estudo pode auxiliar as organizações e os seus gestores de recursos humanos na implementação de práticas que visem à conciliação entre a vida profissional e familiar. Ao mostrar que a facilitação entre o trabalho e a família é o mecanismo por meio do qual estas práticas se relacionam com a satisfação dos empregados, as organizações e os seus gestores ficam a conhecer que, pelo menos em parte, o sucesso da implementação destas práticas depende do quanto os empregados consideram que as mesmas facilitam a sua vida familiar.

\section{Disponibilização de práticas organizacionais de conciliação e satisfação com o trabalho}

Como referido anteriormente, em resposta às mudanças que têm ocorrido na força de trabalho as organizações começaram a implementar determinadas práticas para ajudar os seus trabalhadores assumirem as suas responsabilidades e desenvolverem o seu papel familiar; elas têm sido denominadas práticas de conciliação entre o trabalho e a família (Allen, 2001; Butler, Gasser \& Smart, 2004; Thompson Beauvais \& Lyness, 1999). Podem-se citar como categorias dessas práticas: a flexibilidade de trabalho (por exemplo, a flexibilização de horário, a hipótese de optar por um contrato em tempo parcial ou a hipótese de optar por trabalhar no domicílio); as licenças de trabalho (por exemplo, as licenças maternidade e paternidade ou as licenças de assistência à família); assistência no cuidado de dependentes (por exemplo, disponibilizar creches na organização, atribuir subsídios para assistência às crianças ou estabelecer parcerias com instituições de cuidado de crianças), entre outras (Allen, 2001; Frone, 2003).
Estas práticas facilitam a vida familiar do trabaIhador, ou porque diminuem as exigências do trabalho, ou porque atenuam o efeito dessas exigências na vida familiar. Por exemplo, a utilização de uma licença maternidade ou paternidade facilita a vida familiar do empregado porque, em um momento de elevada exigência familiar, o indivíduo tem licença para não ir trabalhar, permitindo uma dedicação total à família. O mesmo ocorre com a opção por um trabalho em tempo parcial, permitindo ao empregado diminuir o número de horas de trabalho e aumentar o tempo dedicado à família. De um modo distinto, por exemplo, a flexibilidade de horário permite facilitar a vida familiar do empregado, não porque diminui a exigência do trabalho, nomeadamente o número de horas de trabalho, mas porque dá ao empregado a possibilidade de controlar melhor o seu tempo, ajustando as exigências do seu trabalho às exigências da sua família. Ou, de modo semelhante, disponibilizar uma creche no interior da organização não diminui a exigência do trabalho, mas ajuda o empregado a lidar com a exigência familiar de cuidar de filhos pequenos.

Vários estudos revelam que estas práticas conciliadoras têm um efeito positivo nas atitudes dos empregados face à sua atividade profissional. Grover e Crooker (1995) e Thompson et al. (1999) verificaram que os empregados que trabalhavam em organizações que tinham estas práticas de conciliação demonstraram maior compromisso afetivo e menor intenção de deixar a organização. Muse, Harris, Giles e Feild (2008) mostraram que quando as organizações disponibilizavam práticas de conciliação que eram valorizadas pelos empregados, tinham empregados com maior compromisso afetivo. Scandura e Lankau (1997) e Thomas e Ganster (1995) mostraram que o horário flexível se relacionava positivamente com o compromisso afetivo face à organização. Mais interessante, Baltes, Brigss, Huff, Wright e Neuman (1999) desenvolveram um estudo de meta-análise em relação a investigações que testassem os efeitos de intervenções onde tivessem sido introduzidos horários flexíveis e selecionaram somente os estudos que incluíam medidas pré e pós-intervenção. Verificaram que a implementação desta prática de conciliação do trabalho na família promovia a satisfação com o trabalho por parte dos empregados.

Nesta investigação analisou-se o efeito destas práticas de conciliação entre o trabalho e a família na 
satisfação com o trabalho. Esta atitude tem sido a mais investigada nos estudos desenvolvidos na Europa e nos EUA no âmbito do comportamento organizacional e da psicologia do trabalho, e pode ser definida como a avaliação cognitiva e afetiva que o empregado faz acerca do seu trabalho (Brief\&Weiss, 2002; Spector, 1997). Como objetivo central, estes estudos têm procurado analisar em que medida os trabalhadores satisfeitos são também os que exibem comportamentos mais vantajosos para o funcionamento das organizações. De fato, diversificados estudos de meta-análise têm demonstrado o relevo desta atitude para explicar diferentes comportamentos positivos no contexto de trabalho, comprovando que os empregados mais satisfeitos são também aqueles que desempenham melhor as suas tarefas, os que mais exibem comportamentos de cidadania organizacional, os que chegam menos atrasados, os que faltam menos ao trabalho e os que menos abandonam a organização (Harrison, Neuman \& Roth, 2006; Judge, Thoresen, Bono \& Patton, 2001; Riketta, 2008).

Sendo a satisfação com o trabalho uma atitude tão relevante para explicar os comportamentos dos empregados, torna-se crucial conhecer que características organizacionais a promovem. Neste estudo procurou-se analisar em que medida a disponibilização de práticas de conciliação entre o trabalho e a família desempenhava esse papel.

Considerando o que foi referido anteriormente, a seguinte hipótese foi colocada: a disponibilização de práticas de conciliação entre o trabalho e a família por parte das organizações relaciona-se de forma positiva com a satisfação com o trabalho.

\section{Facilitação do trabalho na família como mediador na relação práticas de conciliação e satisfação com o trabalho}

Vários autores salientaram que a natureza da relação entre as práticas de conciliação do trabalho com a família e as atitudes dos empregados continua pouco clara, indicando a necessidade de se desenvolverem mais investigações, que permitam analisar o processo que ocorre na relação entre estas variáveis, isto é, que ajudem a compreender o seu "porque" e o seu "como" (Allen, 2001; Casper \& Buffardi, 2004; Eby, Casper,
Lockwood, Bordeaux \& Brinley, 2005). Por exemplo, Allen (2001) mostrou que as práticas de conciliação entre o trabalho e a família se relacionavam positivamente com a satisfação no trabalho e com o compromisso perante a organização porque aumentavam a percepção de que a organização era um ambiente de trabalho que apoiava a vida familiar dos empregados. Grover e Crooker (1995) sugeriram que estas práticas de conciliação entre o trabalho e a família relacionavam-se positivamente com a ligação afetiva dos empregados em relação à organização porque os empregados consideravam que estas práticas mostravam preocupação da organização para com eles. Bohen e Viveros-Long (1981), por seu lado, sugeriram que o aumento da satisfação com o trabalho positivamente relacionado com a flexibilidade de horário poderia resultar do aumento da percepção de autonomia, assim como do aumento da capacidade para resolver os assuntos familiares.

Estes argumentos sugerem que a relação entre as práticas de conciliação e a satisfação com o trabalho pode ser uma consequência do efeito mediador de outra variável. Nesta investigação considerou-se que F T $\rightarrow F$ poderia ser essa variável. Por um lado, a investigação tem mostrado que $\mathrm{F} T \rightarrow \mathrm{F}$ resulta de características organizacionais que contribuem para a disponibilização de recursos que facilitem o funcionamento do indivíduo na família (Bakker \& Geurts, 2004; Grzywacz, 2002), e as práticas de conciliação podem ser uma dessas características. Por outro lado, outros estudos registraram a existência de uma relação positiva entre $F T \rightarrow F$ e satisfação com o trabalho.

Sieber (1974) e Marks (1977) foram pioneiros ao considerar que a acumulação de papéis poderia trazer benefícios para os indivíduos. No caso específico da facilitação do trabalho na família, considera-se que o trabalho beneficia a família porque os recursos ganhos por meio dele enriquecem o indivíduo e ajudam-no a ter afetos mais positivos e um melhor desempenho na família (Aryee et al., 2005; Barnett \& Hyde, 2001; Boyar \& Mosley, 2007; Butler, Grzywacz, Bass \& Linney, 2005; Greenhaus \& Powell, 2006; Grzywacz \& Marks, 2000; Wayne et al., 2004). Os recursos ganhos por meio da atividade profissional podem facilitar a vida familiar do indivíduo porque promovem o desempenho, reduzem as exigências ou geram recursos adicionais no exercício desse papel (Voydanoff, 2004). 
O trabalho pode fornecer recursos econômicos (salário, assistência à saúde), recursos sociais (suporte social e status social) e recursos psicológicos (sentido de identidade e contribuição pessoal), os quais poderão ser utilizados no desempenho do papel familiar (Grzywacz, 2002). Esta facilitação entre a vida profissional e a familiar pode ser instrumental ou afetiva. No primeiro caso, os recursos gerados no decurso da atividade profissional podem ser transferidos para o desempenho do papel familiar: por exemplo, com os benefícios de assistência à saúde ganhos na empresa em que trabalha, o indivíduo pode garantir melhor bem-estar para a sua família. No segundo caso, os recursos ganhos no decurso da atividade profissional podem gerar um afeto positivo que se transfere para o desempenho do papel familiar: uma promoção profissional, por exemplo, pode trazer uma satisfação ao indivíduo que se traduz em maior disponibilidade e boa disposição no exercício da sua função familiar. Quer em uma situação ou em outra, os recursos ganhos no contexto profissional permitem ao indivíduo ter afetos mais positivos e um melhor desempenho no seu papel familiar (Greenhaus \& Powell, 2006).

As práticas de conciliação entre o trabalho e a família podem ser vistas como formas específicas de disponibilizar esses recursos e desenvolver esses mecanismos de facilitação entre o trabalho e a família. Concretamente, as práticas de flexibilidade (horário, local de trabalho) associam-se a um aumento da autonomia e controle por parte do empregado (Thomas \& Ganster, 1995), implicando competências de resolução de problemas e julgamentos acerca de necessidades divergentes no trabalho, as quais poderão ajudar o empregado a planejar as suas tarefas familiares e executá-las com eficácia (Grzywacz \& Butler, 2005). No caso das práticas de assistência a dependentes, ao ajudar o empregado a assegurar as suas responsabilidades familiares pode-se permitir a ele uma maior dedicação ao trabalho, que lhe dará maiores oportunidades de desenvolvimento e melhores níveis de desempenho, contribuindo para promover a sua autoestima e confiança, que podem ser usadas para aumentar os afetos positivos e o desempenho na família (Aryee et al., 2005; Greenhaus \& Parasuraman, 1999).
Por outro lado, como referido anteriormente, Carlson et al. (2006) defenderam que os indivíduos que registrassem elevados níveis de facilitação do trabalho na família experimentariam elevada satisfação com o trabalho, e vários estudos empíricos têm comprovado essa relação. Por exemplo, Wayne et al. (2004) verificaram que a $\mathrm{F} T \rightarrow \mathrm{F}$ relacionava-se positivamente com a satisfação com o trabalho e com o esforço colocado neste domínio. Aryee et al. (2005), em um estudo realizado na Índia, verificaram esta relação e consideraram que ela poderia ocorrer porque a $\mathrm{FT} \rightarrow \mathrm{F}$ poderia melhorar o desempenho dos indivíduos, o que conduziria ao aumento da sua autoestima e ao ganho de determinadas recompensas que, posteriormente, originariam maior satisfação com o trabalho.

Considerando a revisão de literatura realizada, que demonstra que as práticas organizacionais de conciliação entre o trabalho e a família relacionam-se positivamente com a F $\rightarrow \mathrm{F}$ e que, por outro lado, esta $\mathrm{FT} \rightarrow \mathrm{F}$ apresenta uma relação positiva com a satisfação com o trabalho, colocou-se uma segunda hipótese: a facilitação do trabalho na família medeia a relação entre as práticas de conciliação do trabalho e da família e a satisfação com o trabalho.

Estas hipóteses foram testadas em um estudo de campo no qual trabalhadores de diferentes profissões e organizações responderam a um questionário.

\section{Método}

\section{Participantes}

Participaram deste estudo 342 indivíduos, com as seguintes características demográficas: 171 (50\%) do sexo feminino, idade média de 37,4 anos (desvio-padrão - DP=9,72), escolaridade média ao nível do ensino secundário e média do número de filhos de 1,28 $(\mathrm{DP}=1,20)$

Os indivíduos tinham diferentes profissões, por exemplo, operários, trabalhadores do comércio, vendedores e professores. Trabalhavam em organizações com características distintas e de diferentes ramos de atividade, ou seja, empresas de grandes ou pequenas dimensões, companhias de seguros, entidades bancárias, administração pública e fábricas. 


\section{Medidas}

Práticas de conciliação do trabalho e da família: utilizou-se uma adaptação da listagem de práticas anteriormente utilizada por Allen (2001). Esta lista continha dez práticas, agrupadas em duas categorias: a) práticas de flexibilidade - horário de trabalho flexível, semana de trabalho comprimida (as mesmas horas de trabalho semanal em menos dias), trabalho a partir de casa ou teletrabalho e trabalho em tempo parcial; b) práticas de suporte no cuidado de dependentes - creches/jardins de infância no local de trabalho, subsídios de apoio à infância, serviços de informação/encaminhamento sobre creches/jardins de infância, licença maternidade paga, licença paternidade paga e cuidado de idosos. Foram retiradas as licenças maternidade e paternidade porque estas duas práticas de conciliação são obrigatórias na lei portuguesa, não podendo, por isso, ser utilizadas para diferenciar os participantes.

Cada participante devia assinalar se cada uma das práticas estava disponível na organização na qual trabalhava, registrando 0 se a prática não existisse e 1 se estivesse disponível. Para cada participante foi calculada a média de práticas de conciliação disponibilizadas pela organização na qual trabalhava.

Satisfação com o trabalho: utilizou-se uma versão abreviada de cinco itens da escala de Brayfield e Rothe (1951), à semelhança do efetuado por Aryee et al. (2005). Exemplos de itens utilizados são: "Sinto-me bastante satisfeito(a) com a minha vida profissional" e "Encontro uma verdadeira satisfação no meu trabalho". Os indivíduos davam as suas respostas em uma escala tipo Likert de 5 pontos, variando de discordo muito (1) a concordo muito (5). Resultados elevados na escala correspondem a um maior grau de satisfação com o trabalho. componentes principais, com rotação ortogonal dos eixos. Esta análise demonstrou a existência de apenas um fator, que explica $62,77 \%$ da variância dos resultados. Além disso, verificou-se que os itens utilizados para avaliar esta variável obtiveram uma consistência interna de $\alpha=0,84$.

Facilitação do trabalho na família: utilizou-se a dimensão da facilitação do trabalho na família desenvolvida por Carlson et al. (2006). Esta medida possui um total de nove itens e consiste de três áreas de facilitação (desenvolvimento, afeto e capital), cada uma com três itens. Exemplos dos itens são: "O meu envolvimento com o meu trabalho ajuda-me a compreender diferentes pontos de vista e isto me ajuda a ser um melhor membro da minha família", "O meu envolvimento com o meu trabalho põe-me com bom humor e isto me ajuda a ser um melhor membro da minha família", "O meu envolvimento com o meu trabalho ajuda-me a sentir preenchido(a) como pessoa e isto me ajuda a ser um melhor membro da minha família". Os indivíduos assinalavam o grau de acordo com cada uma das afirmações, usando uma escala de 5 pontos, de discordo fortemente (1) a concordo fortemente (5). As instruções estabeleciam claramente que os sujeitos deviam concordar com a totalidade da frase, ou seja, tinham que determinar se o ganho ocorria e se conduzia a um melhor funcionamento.

Realizou-se uma análise fatorial exploratória em componentes principais, com rotação ortogonal dos eixos, que demonstrou a existência de um fator, ao contrário dos três fatores pressupostos pelos autores. Foram eliminados dois itens por terem se revelados ambíguos. O fator explica 60,47\% da variância dos resultados e os itens que o compõem revelaram uma consistência interna de $\alpha=0,90$.

Variáveis controle: alguns autores têm verificado que determinadas variáveis, como o sexo, a idade, o número de filhos e a escolaridade têm influência na facilitação do trabalho na família e na satisfação com o trabalho (Behson, 2002; Clark, 1997; Clark, 2001; Grzywacz \& Marks, 2000; Premeaux, Adkins \& Mossholder, 2007; Wayne et al., 2004). Por esta razão, apesar de tais variáveis não terem sido colocadas nas hipóteses, seus efeitos foram controlados de modo a não enviesarem os resultados. A variável sexo foi avaliada como variável dummy (mulheres $=0$; homens $=1$ ). Por outro lado, a variável escolaridade foi codificada como 1 para as habilitações ao nível do ensino básico, 2 para o ensino secundário, 3 para a licenciatura e 4 para o mestrado/doutoramento. A idade dos participantes foi registrada em anos.

\section{Procedimentos}

Os investigadores contataram pessoalmente os indivíduos e explicaram o objetivo geral do estudo, isto 
é, estudar a relação entre o trabalho e a família, referindo que o importante era a opinião pessoal de cada um sobre os assuntos abordados, não existindo, por isso, respostas certas ou erradas. Foi igualmente referido que o questionário era anônimo e que a confidencialidade dos dados individuais estava assegurada. Depois de os indivíduos confirmarem a sua disponibilidade e interesse em participar do estudo, os investigadores forneceram o questionário e o respectivo envelope, no qual devia ser colocado o questionário depois de devidamente preenchido. Cada questionário foi respondido individualmente por cada um dos participantes.

\section{Resultados}

Ao analisar a Tabela 1 é possível verificar que as habilitações literárias apresentam uma correlação significativa e positiva, embora fraca, com a satisfação com o trabalho $(r=0,16, p<0,01)$. A existência desta correlação confirma a importância de controlar esta variável. Em relação às variáveis estudadas, verifica-se que a disponibilização de práticas organizacionais conciliadoras dos domínios do trabalho e da família correlacionou-se positivamente de forma significativa, embora de um modo fraco, com a satisfação com o trabalho $(r=0,12, p<0,05)$ e com a FT $\rightarrow F(r=0,13, p<0,05)$. Além destas correlações, verifica-se ainda que a F T $\rightarrow F$ correlacionou-se de forma significativa e positiva com a satisfação com o trabalho, sendo esta uma correlação forte $(r=0,56, p<0,01)$

Na sequência foram realizadas análise de regressões hierárquicas para averiguar as hipóteses do estudo, uma vez que este método permite controlar o efeito que outras variáveis podem eventualmente ter na relação entre as variáveis estudadas. Deste modo, em um primeiro passo foram introduzidas as variáveis controle (idade, sexo, número de filhos e escolaridade) e, em um segundo passo, introduziu-se a variável preditora, consoante a hipótese.

Em relação ao efeito das práticas de conciliação na satisfação com o trabalho (hipótese 1), os resultados permitem suportar esta hipótese. De fato, como se mostra na Tabela 2, verificou-se que as variáveis controle (modelo 1) explicam 3,7\% da variância dos resultados, e que 1,2\% da satisfação com o trabalho deve-se à disponibilização de práticas. O modelo 2 explica, no total, 4,9\% da variância nos resultados. Éainda de referir, ao analisar os coeficientes de regressão, que o efeito que a disponibilização de práticas tem na satisfação com o trabalho é significativamente positivo, embora fraco $(\beta=0,111, p<0,05)$.

A hipótese 2 sugeria que a $\mathrm{FT} \rightarrow \mathrm{F}$ iria mediar a relação entre as práticas de conciliação do trabalho e da família e a satisfação com o trabalho. De acordo com Baron \& Kenny (1986), a hipótese de mediação só pode ser testada se forem satisfeitas três condições: primeiro, a variável preditora deve afetar a variável mediadora; segundo, a variável preditora deve afetar a variável critério; e terceiro, a variável mediadora deve afetar a variável critério. Além disto, cada uma destas três condições deve se revelar significativa na direção prevista.

A segunda condição corresponde à hipótese 1 deste estudo e, como já referido, esta hipótese foi suportada. Deste modo, fez-se necessário verificar se as restantes duas condições foram ou não satisfeitas. Em

Tabela 1. Médias (M), desvios-padrão (DP)e coeficientes de correlação das variáveis estudadas. Lisboa (Portugal), 2008.

\begin{tabular}{|c|c|c|c|c|c|c|c|c|}
\hline Variáveis & M & DP & 1 & 2 & 3 & 4 & 5 & 6 \\
\hline 1. Idade & 37,40 & 9,72 & & & & & & \\
\hline 2. Sexo & 0,50 & 0,50 & $0,11^{*}$ & & & & & \\
\hline 3. Número de filhos & 1,28 & 1,21 & $0,25^{* *}$ & $-0,04$ & & & & \\
\hline 4. Escolaridade & 2,07 & 0,70 & $-0,11^{*}$ & $-0,10$ & $-0,15^{* *}$ & & & \\
\hline 5. Satisfação com o trabalho & 3,37 & 0,70 & 0,00 & 0,03 & 0,05 & $0,16^{* *}$ & & \\
\hline 6. Práticas de conciliação & 1,80 & 1,61 & $-0,04$ & $-0,09$ & $-0,03$ & 0,05 & $0,12^{* *}$ & \\
\hline 7. $\mathrm{FT} \rightarrow \mathrm{F}$ & 3,37 & 0,65 & 0,09 & $-0,01$ & $-0,06$ & 0,08 & $0,56^{* *}$ & $0,13^{*}$ \\
\hline
\end{tabular}

${ }^{*} p<0,05 ;{ }^{* *} p<0,01 ; F T \rightarrow F$ : facilitação do trabalho em família. 
Tabela 2. Resultados das análises de regressões hierárquicas. Lisboa (Portugal), 2008.

\begin{tabular}{|c|c|c|c|c|c|c|c|c|}
\hline \multirow{3}{*}{ Variáveis } & \multicolumn{8}{|c|}{ Coeficientes padronizados de regressão (Beta) } \\
\hline & \multicolumn{2}{|c|}{$\begin{array}{c}\text { Satisfação com o } \\
\text { trabalho }\end{array}$} & \multicolumn{2}{|c|}{$\mathrm{FT} \rightarrow \mathrm{F}$} & \multicolumn{2}{|c|}{$\begin{array}{c}\text { Satisfação com o } \\
\text { trabalho }\end{array}$} & \multicolumn{2}{|c|}{$\begin{array}{c}\text { Satisfação com o } \\
\text { trabalho }\end{array}$} \\
\hline & Modelo 1 & Modelo 2 & Modelo 1 & Modelo 2 & Modelo 1 & Modelo 2 & Modelo 1 & Modelo 2 \\
\hline Idade & $-0,029$ & $-0,025$ & 0,104 & 0,110 & $-0,033$ & $-0,092$ & $-0,033$ & $-0,088$ \\
\hline Sexo & 0,059 & 0,067 & $-0,009$ & 0,001 & 0,061 & 0,064 & 0,061 & 0,069 \\
\hline Número de filhos & 0,085 & 0,087 & $-0,070$ & $-0,067$ & 0,086 & $0,127^{* *}$ & 0,086 & $0,128^{* *}$ \\
\hline Escolaridade & $0,182^{* *}$ & $0,177^{* *}$ & 0,076 & 0,069 & $0,172^{* *}$ & $0,126^{* *}$ & $0,172^{* *}$ & $0,122^{* *}$ \\
\hline Práticas de conciliação & & $0,111^{*}$ & & $0,125^{*}$ & & & & 0,072 \\
\hline $\mathrm{FT} \rightarrow \mathrm{F}$ & & & & & & $0,573^{* * *}$ & & $0,564^{* * *}$ \\
\hline $\mathrm{F}$ & $3,070^{*}$ & $3,300^{* *}$ & 1,450 & 2,200 & $2,840^{*}$ & $35,110^{* * *}$ & $2,84^{*}$ & $29,81^{* * *}$ \\
\hline$R^{2}$ Ajustado & 0,025 & 0,034 & 0,005 & 0,018 & 0,022 & 0,346 & 0,022 & 0,349 \\
\hline Mudança $R^{2}$ & $0,037^{*}$ & $0,012^{*}$ & 0,018 & $0,016^{*}$ & $0,340^{*}$ & $0,322^{* * *}$ & $0,34^{*}$ & $0,327^{* * *}$ \\
\hline
\end{tabular}

$n=342 ; \mathrm{F} \mathrm{T} \rightarrow \mathrm{F}=$ facilitação do trabalho na família; ${ }^{*} p<0,05 ;{ }^{* *} p<0,01 ;{ }^{* *} p<0,001$.

relação à primeira condição, ao analisar a Tabela 2 verifica-se que a as práticas de conciliação explicam 1,6\% da variância dos resultados na $F T \rightarrow F$, sendo que, no total, o modelo 2 explica 3,3\%. Por outro lado, verifica-se que o efeito que estas práticas conciliadoras têm na $\mathrm{F} T \rightarrow \mathrm{F}$ é positivo e relativamente fraco, mas significativo $(\beta=0,125, p<0,05)$. Estes dados permitem concluir que a primeira condição está igualmente satisfeita.

Do mesmo modo, a terceira condição também está satisfeita. De fato, ao analisar a Tabela 2 verifica-se que a introdução da variável $\mathrm{FT} \rightarrow \mathrm{F}$ acrescentou 32,2\% para explicar a variância dos resultados na satisfação com o trabalho. No total, o modelo 2 explica 35,6\% dessa mesma variância. Além disso, verifica-se que o efeito que a $\mathrm{F} T \rightarrow \mathrm{F}$ tem na satisfação com o trabalho é significativamente positivo e forte $(\beta=0,573, p<0,001)$.

Uma vez que as três condições necessárias para se testar a hipótese de mediação foram satisfeitas, foi possível analisar a hipótese 2. Como demonstrado pela Tabela 2, as variáveis controle (modelo 1) explicam 3,4\% da variância dos resultados, enquanto a disponibilização de práticas organizacionais de conciliação e a F T $\rightarrow F$ acrescentam $32,7 \%$ para explicar a variância dos resultados na satisfação com o trabalho. No total, o modelo 2 explica 36,1\% dessa mesma variância. Ao analisar os coeficientes de regressão, verifica-se que, apesar de anteriormente as práticas de conciliação terem apresentado um efeito significativamente positivo na satisfação com o trabalho (hipótese 1), a introdução da variável $F T \rightarrow F$ na mesma equação fez com que o efeito desta variável deixasse de ser significativo. Além disso, esta análise de regressão demonstrou que a FT $\rightarrow$ F tem um efeito significativamente positivo e forte na satisfação com o trabalho $(\beta=0,564, p<0,001)$. Estes resultados suportam a hipótese 2 , que propunha que a $F \mathrm{~T} \rightarrow \mathrm{F}$ mediava a relação entre as práticas de conciliação e a satisfação com o trabalho. É ainda de referir que, uma vez que na presença da $\mathrm{FT} \rightarrow \mathrm{F}$ as práticas de conciliação deixam de exercer efeito na satisfação com o trabalho, esta mediação é total. Em suma, pode-se concluir que existe uma relação entre as práticas de conciliação e a satisfação com o trabalho porque estas práticas estão relacionadas com aspectos do trabalho que facilitam a vida familiar, fazendo com que os trabalhadores considerem que existe $\mathrm{FT} \rightarrow \mathrm{F}$.

\section{Discussão}

Os resultados obtidos neste estudo suportam as hipóteses colocadas. Mais especificamente, os resultados demonstram que o fato de a organização disponibilizar práticas de conciliação entre o trabalho e a família é uma forma de conseguir ter trabalhadores mais satisfeitos. Mais interessante, com este estudo confirmou-se que esta relação ocorria porque tais práticas de conciliação eram vistas pelos empregados como uma forma de facilitação do trabalho na família.

Conforme o esperado, o fato de as organizações, por exemplo, praticarem horários flexíveis, semanas de trabalho comprimidas e desenvolverem programas de 
apoio à assistência a dependentes relacionou-se positivamente com a satisfação no trabalho por parte dos seus empregados. No entanto, os dados demonstraram que apesar das práticas de conciliação terem uma relação positiva com a satisfação no trabalho, esse efeito é pequeno. Uma possível explicação para este efeito relativamente pequeno pode residir no fato de os participantes do estudo se encontrarem a trabalhar em organizações que disponibilizam poucas dessas práticas. Ou seja, verificou-se que a média de práticas de conciliação que as organizações disponibilizam é muito baixa, o que pode ter influenciado os resultados relativos ao efeito que esta disponibilização tem na satisfação com o trabalho. Esta média baixa relativamente ao número de práticas conciliadoras que as organizações disponibilizam reflete a realidade da grande maioria das organizações em Portugal. Deste modo, seria interessante, à semelhança do efetuado por Muse et al. (2008), selecionar organizações que efetivamente disponibilizam mais práticas de conciliação e analisar a sua relação com a satisfação no trabalho.

Por outro lado, os dados deste estudo demonstraram, conforme se esperava, que a relação entre estas práticas de conciliação e a satisfação com o trabalho é mediada pela F T $\rightarrow$ F. Assim, é possível afirmar que a disponibilização de práticas só tem efeito na satisfação com o trabalho quando os trabalhadores consideram que a sua participação no papel de trabalho Ihes fornece "experiências, aptidões e oportunidades" (Frone, 2003, p.145) que facilitam a sua participação no papel familiar. Isto é, para que os trabalhadores se sintam satisfeitos com o seu trabalho, não basta que as organizações disponibilizem práticas conciliadoras dos domínios do trabalho e da família; é necessário que os trabalhadores considerem que essas práticas Ihes facilitam a relação entre as suas vidas profissional e familiar. Como referido por outros autores, as práticas de conciliação permitem aos trabalhadores desenvolverem competências que podem ser úteis no desempenho do seu papel na família (Grzywacz \& Butler, 2005), assim como Ihes podem dar maiores oportunidades de desenvolvimento e melhores níveis de desempenho no trabalho, contribuindo para promover a sua autoestima e confiança, que podem ser usadas para aumentar os afetos positivos e o desempenho na família (Aryee et al., 2005; Greenhaus \& Parasuraman, 1999).

Os dados desta investigação, ao salientarem o papel mediador da F $\mathrm{T} \rightarrow \mathrm{F}$ na relação entre as práticas de conciliação e a satisfação com o trabalho, estão em conformidade com outros estudos, que demonstraram que, embora estas práticas possam servir como ferramentas valiosas para ajudar os trabalhadores a gerirem o seu papel no trabalho e o seu papel na família, a simples implementação destas práticas não parece ser suficiente (Allen, 2001; Shockley \& Allen, 2001; Thompson et al., 1999). Assim, este estudo fornece uma explicação sobre o mecanismo pelo qual práticas conciliadoras têm efeito em uma atitude importante dos trabalhadores: a sua satisfação com o trabalho. Deste modo, a pesquisa assume especial importância porque são poucos os investigadores que se têm focado na identificação dos processos que explicam como e porque as práticas de conciliação entre o trabalho e a família têm um impacto positivo nos empregados (Allen, 2001).

Existem algumas limitações do estudo descrito que devem ser consideradas. Primeiro, os dados foram recolhidos exclusivamente por meio de um questionário ao qual os sujeitos tinham que responder, ou seja, foi utilizada uma medida de autorrelato aplicada em um único período de tempo. Assim, as respostas dos sujeitos podem refletir, por exemplo, o seu estado emocional daquele momento e não a sua verdadeira opinião sobre os temas abordados. Por outro lado, pode acontecer que os trabalhadores não estejam completamente conscientes das práticas que são disponibilizadas nas organizações onde trabalham. Por estas e outras razões, esta forma de recolhimento de dados pode fazer com que surjam determinados enviesamentos nos resultados. Uma forma de reduzir a probabilidade de ocorrer estes enviesamentos é desenvolver estudos longitudinais futuros que permitam fazer avaliações repetidas no decurso do tempo.

Uma segunda limitação deste estudo relaciona-se com o fato de as práticas disponibilizadas pelas organizações não terem sido agrupadas em categorias, mas estudadas enquanto um único conjunto. No seu estudo, Allen (2001) agrupou a lista de benefícios em duas categorias: benefícios relacionados com o trabalho flexível (horário flexível e semana de trabalho compri- 
mida) e suporte relacionado com o cuidado de dependentes (creches/jardins de infância no local de trabalho e apoio nos cuidados a idosos). De fato, os seus resultados demonstraram que apenas a categoria do trabalho flexível esteve significativamente relacionada com as variáveis estudadas. Allen (2001) refere que estas descobertas podem ocorrer porque todos os empregados podem se beneficiar de opções de trabalho flexível, enquanto é mais provável que os benefícios relacionados ao cuidado de dependentes sejam valorizados apenas por um subconjunto da força de trabalho. Assim, uma vez que diferentes tipos de práticas têm diferentes relações com outras variáveis, seria importante analisar as hipóteses deste estudo utilizando categorias de práticas em vez de agrupá-las em um único conjunto.

Outra limitação inerente a este estudo reside no fato de se estar a lidar, indiretamente - por meio da participação dos sujeitos - com muitas organizações. Estas, por sua vez, podem diferir substancialmente umas das outras em termos de, por exemplo, clima, cultura suporte das chefias e tantas outras dimensões que não foram controladas nesta investigação. Por isso, aspectos relacionados a estas dimensões podem ter exercido algum efeito nas variáveis estudadas.

Vários estudos têm demonstrado a importância da satisfação com o trabalho, porque os trabalhadores mais satisfeitos são também os que atingem melhores níveis de desempenho, os que mais exibem comportamentos de cidadania organizacional, os que menos faltam, os que menos chegam atrasados e os que menos abandonam a organização (Harrison et al., 2006; Judge et al., 2001; Riketta, 2008). Deste modo, parece extremamente importante para o bom funcionamento das organizações que estas consigam fazer com que seus trabalhadores estejam satisfeitos com o trabalho. Este estudo, ao salientar a importância da F T $\rightarrow$ F enquanto variável mediadora da relação entre as práticas de conciliação e a satisfação com o trabalho, sugere que uma forma que as organizações têm para fazerem com que seus trabalhadores estejam satisfeitos com o trabalho é disponibilizar práticas conciliadoras dos domínios do trabalho e da família que sejam percebidas pelos trabalhadores como uma forma de o desempenho do papel profissional enriquecer o desempenho do papel familiar. Isto significa, como já referido anteriormente, que não basta às organizações disponibilizarem práticas, mas que essas mesmas práticas têm que ser valorizadas pelos trabalhadores enquanto práticas que Ihes permitem desenvolver competências e atitudes que são úteis para o desempenho do seu papel familiar. Esta conclusão tem importantes implicações para as organizações. Assim, em conformidade com o estudo de Muse et al. (2008), estes dados sugerem que, quando do momento de decisão sobre as práticas que devem ou não ser disponibilizadas, as organizações devem avaliar as percepções que os trabalhadores têm sobre o valor das práticas que se pretende disponibilizar.

Os dados obtidos salientam o papel importante da $\mathrm{F} \mathrm{T \rightarrow F} \mathrm{e,} \mathrm{consequentemente,} \mathrm{a} \mathrm{importância} \mathrm{de} \mathrm{as}$ organizações proporcionarem aos seus colaboradores uma vida mais equilibrada entre trabalho e família. Mais especificamente, Thompson et al. (1999) afirmaram que, se as organizações não proporcionarem tal equilíbrio, surgirão tensões na vida pessoal dos colaboradores que, por sua vez, afetarão a capacidade dos empregados de se concentrarem e serem produtivos e criativos no trabalho.

\section{Referências}

Allen, T. D. (2001). Family-supportive work environments: the role of organizational perceptions. Journal of Vocational Behavior, 58 (3), 414-435.

Aryee, S., Srinivas, E. S., \& Tan, H. H. (2005). Rhythms of life: antecedents and outcomes of work-family balance in employed parents. Journal of Applied Psychology, 90 (1), 132-146.

Bakker, A. B., \& Geurts, S. A. E. (2004). Towards a dual-process model of work-home interference. Work and Occupations, 31 (3), 345-366.

Baltes, B. B., Briggs, T. E., Huff, J. W., Wright, J. A., \& Neuman, G. A. (1999). Flexible and compressed workweek schedules: a meta-analysis of their effects on work-related criteria. Journal of Applied Psychology, 84 (4), 496-513.

Barnett, R. C., \& Hyde, J. S. (2001). Women, men, work, and family. American Psychologist, 56 (10), 781-796.

Baron, R. M., \& Kenny, D. A (1986). The moderator-mediator variable distinction in social psychology research: conceptual, strategic, and statistical considerations. Journal of Personality and Social Psychology, 51 (6), 1173-1182.

Behson, S. J. (2002). Which dominates? The relative importance of work-family organizational support and 
general organizational context on employee outcomes. Journal of Vocational Behavior, 61 (1), 53-72.

Bohen, H. H., \& Viveros-Long, A. (1981). Balancing jobs and family life: do flexible schedules really help? Philadelphia: Temple University Press.

Boyar, S. L., \& Mosley, D. C. (2007). The relationship between core self-evaluations and work and family satisfaction: the mediating role of work-family conflict and facilitation. Journal of Vocational Behavior, 71 (2), 265-281.

Brayfield, A. H., \& Rothe, H. F. (1951). An index of job satisfaction. Journal of Applied Psychology, 35 (5), 307-311.

Brief, A. P., \& Weiss, H. M. (2002). Affect in the workplace. Annual Review of Psychology, 53 (1), 279-307.

Butler, A., Gasser, M., \& Smart, L. (2004). A social-cognitive perspective on using family-friendly benefits. Journal of Vocational Behavior, 65 (1), 57-70.

Butler, A. B., Grzywacz, J. G., Bass, B. L., \& Linney, K. D. (2005). Extending the demands-control model: a dialy diary study of Job characteristics, work-family conflict and work-family facilitation. Journal of Occupational and Organizational Psychology, 78 (1), 155-169.

Carlson, D., Kacmar, K., Wayne, J., \& Grzywacz, J. (2006). Measuring the positive side of the work-family interface: development and validation of a work-family enrichment scale. Journal of Vocational Behavior, 68 (1), 131-164.

Casper, W. J., \& Buffardi, L. C. (2004). Work-life benefits and job pursuit intention: the role of anticipated organizational support. Journal of Vocational Behavior, 65 (3), 391-410.

Clark, A. E. (1997). Job satisfaction and gender: why are women so happy at work? Labour Economics, 4 (4), 341-372.

Clark, S. C. (2001). Work cultures and work/family balance. Journal of Vocational Behavior, 58 (3), 348-365.

Eby, L., Casper, W., Lockwood, A., Bordeaux, C., \& Brinley, A. (2005). Work and family research in IO/OB: content analysis and review of the literature (1980-2002). Journal of Vocational Behavior, 66 (1), 124-197.

Ford, M. T., Heinen, B. A., \& Langkamer, K. L. (2007). Work and family satisfaction and conflict: a meta-analysis of cross-domain relations. Journal of Applied Psychology, 92 (1), 57-80.

Frone, M. R. (2003). Work-family balance. In J. C. Quick \& L. E. Tetrick (Eds.), Handbook of occupational health psychology (pp.143-162). Washington, DC: APA.

Greenhaus, J., Allen, T. D., \& Spector, P. (2006). Health consequences of work-family conflict: the dark side of the work-family interface. Research in Occupational Stress and Well Being. Employee Health, Coping and Methodologies, 5, 61-98.

Greenhaus, J. H., \& Beutell, N. J. (1985). Sources of conflict between work and family roles. Academy of Management Review, 10 (1), 76-88.
Greenhaus, J. H., \& Parasuraman, S. (1999). Research on work, family, and gender: current status and future directions. In G. N. Powell (Ed.), Handbook of gender and work (pp.391-412). Newbury Park, CA: Sage.

Greenhaus, J. H., \& Powell, G. N. (2006). When work and family are allies: a theory of work-family enrichment. Academy of Management Review, 31 (1), 72-92.

Grover, S. L., \& Crooker, K. J. (1995). Who appreciates family-responsive human resource policies: the impact of family-friendly policies on the organizational attachment of parents and non-parents. Personnel Psychology, 48 (2), 271-288.

Grzywacz, J. G. (2002). Toward a theory of work-family facilitation. Paper presented at the 32nd Annual Theory Construction and Research Methodology Workshop of the Persons; National Council on Family Relations, Houston, TX.

Grzywacz, J. G., \& Butler, A. B. (2005). The impact of job characteristics on work-to-family facilitation: testing a theory and distinguishing a construct. Journal of Occupational Health Psychology, 10 (1), 97-109.

Grzywacz, J. G., \& Marks, N. F. (2000). Reconceptualizing the work-family interface: an ecological perspective on the correlates of positive and negative spillover between work and family. Journal of Occupational Health Psychology, 5 (1), 111-126.

Harrison, D. A., Neuman, D. A., \& Roth, P. L. (2006). How important are job attitudes? A meta-analytic comparisons of integrative behavioural outcomes and time sequences. Academy of Management Review, 49 (2), 305-325.

Judge, T. A., Thoresen, C. J., Bono, J. E., \& Patton, G. K. (2001). The job satisfaction-job performance relationship: a qualitative and quantitative review. Psychological Bulletin, 127 (3), 376-407.

Marks, S. R. (1977). Multiple roles and role strain: some notes on human energy, time and commitment. American Sociological Review, 42 (6), 921-936.

Muse, L., Harris, S. G., Giles, W. F., \& Field, H. S. (2008). Work-life benefits and positive organizational behavior: is there a connection? Journal of Organizational Behavior, 29 (2), 171-192.

Parasuraman, S., \& Greenhaus, J. H. (2002). Toward reducing some critical gaps in work-family research. Human Resource Management Review, 12 (3), 299-312.

Perrewé, P., \& Hochwarter, W. (2001). Can we really have it all? The attainment of work and family values. Current Directions in Psychological Science, 10 (1), 29-33.

Premeaux, S. F., Adkins, C. L., \& Mossholder, K. W. (2007). Balancing work and family: a field study of multi-dimensional, multi-role work-family conflict. Journal of Organizational Behavior, 28 (6), 705-727.

Riketta, M. (2008). The causal relation between job attitudes and performance: a meta-analysis of panel studies. Journal of Applied Psychology, 93 (2), 472-481.

Scandura, T., \& Lankau, M. (1997). Relationships of gender, family responsabiliy and flexible work hours to 
organizational commitment and job satisfaction. Journal of Organizational Behavior, 18 (4), 377-391.

Seligman, M. E. P. (2002). Authentic happiness: using the new positive psychology to realize your potential for lasting fulfilment. New York: Free Press.

Shockley, K. M., \& Allen, T. D. (2007). When flexibility helps: another look at the availability of flexible work arrangements and work-family conflict. Journal of Vocational Behavior, 71 (3), 479-493.

Sieber, S. D. (1974). Toward a theory of role accumulation. American Sociological Review, 39 (4), 567-578.

Spector, P. E. (1997). Job satisfaction: application assessment, cause and consequences. London: Sage.

Thomas, L. T., \& Ganster, D. C. (1995). Impact of family-supportive work variables on work-family conflict and strain: a control perspective. Journal of Applied Psychology, $80(1), 6-15$.
Thompson, C. A., Beauvais, L. L., \& Lyness, K. S. (1999). When work-family benefits are not enough: the influence of work-family culture on benefit utilization, organizational attachment, and work-family conflict. Journal of Vocational Behavior, 54 (3), 392-415.

Voydanoff, P. (2004). Implications of work and community demands and resources for work-to-family conflict and facilitation. Journal of Occupational Health Psychology, 9 (4), 275-285.

Wayne, J., H., Musisca, N., \& Fleeson, W. (2004). Considering the role of personality in the work-family experience: relationships of the big five to work-family conflict and facilitation. Journal of Vocational Behavior, 64 (1), 108-130.

Recebido em: 21/8/2008

Versão final reapresentada em: 6/3/2009

Aprovado em: 8/5/2009 